\title{
Ionospheric propagation effects on spectral widths measured by SuperDARN HF radars
}

\author{
X. Vallières, J.-P. Villain, C. Hanuise, and R. André \\ LPCE/CNRS, 3A Av. de la Recherche Scientifique, 45071 Orléans Cedex 2, France \\ Received: 25 September 2003 - Revised: 24 February 2004 - Accepted: 1 March 2004 - Published: 14 June 2004
}

\begin{abstract}
SuperDARN HF radars provide a global survey of the large-scale convection transversely to the Earth's magnetic field in the high-latitude ionosphere. In addition to the mean plasma velocity, this network also provides measurements of spectral widths which are related to the level of turbulence of the sounded plasma. There is an increasing interest in using spectral widths in geophysical studies, since they are used to monitor the footprints of several magnetospheric regions. In the present paper, we show the effect of radio wave propagation through a typical turbulent ionosphere on spectral widths measured by SuperDARN radars. This effect has already been evidenced experimentally in a previous paper. Here, we model the effects of meso-scale structures on a radar wave front and study their impact on a typical measurement. Numerical simulations reproduce the effect evidenced experimentally and show the role of meso-scale structures $(1-10 \mathrm{~km})$ in the systematic bias that affects spectral width values. As in experimental data, this effect is shown to be increasing with decreasing radar frequency.
\end{abstract}

Key words. Ionosphere (auroral ionosphere; wave propagation) - Radio science (ionospheric propagation)

\section{Introduction}

In studying the Sun-Earth connection, the spectral width of ionospheric radar echoes measured by SuperDARN (Super Dual Auroral Radar Network) is a parameter of importance, since it reveals the level of turbulence of the high-latitude ionosphere. This parameter finds applications in localizing the ionospheric footprints of magnetospheric regions and is useful to understand the physical processes governing energy exchanges within the magnetosphere.

In a previous paper, Vallières et al. (2003) have evidenced a clear effect in the range-frequency distribution of spectral widths measured in the high-latitude ionosphere by coher-

Correspondence to: J.-P. Villain

(jvillain@cnrs-orleans.fr) ent HF radars from the SuperDARN network. Conducting statistical studies over several data sets, the authors of this paper, which will now be referred to as P1, have shown an increase of the spectral width with range which is related to the sounding radar frequency. This effect has been related to ionospheric radio wave propagation and shown to be independent of geophysical conditions and radar parameters. As discussed in P1, this increase is related to a loss of coherence in the wave front as it propagates to the scattering zones through a turbulent ionosphere.

It is a well-known feature of high-latitude ionospheric propagation that the amplitude and the phase of waves propagating through the ionosphere experience fluctuations due to structures at various scales of the electron density resulting from exchange processes between the Earth's ionosphere and the magnetosphere or the interplanetary medium. Several methods exist to model and take these fluctuations into account, depending on the geometry and on the wavelength to the inhomogeneity scale ratio. The characteristic scale of electron density inhomogeneities encountered along the wave path plays a major role in the resolution of such problems. Indeed, large-scales of several hundreds of $\mathrm{km}$ are known to deflect waves from their initial direction, leading to refraction effects. On the contrary, micro-scales, of the order of the wavelength or lower, produce diffusion. In the configuration of SuperDARN radars, irregularities with a characteristic size equal to the half wavelength generate the echo through coherent backscattering. Meso-scale irregularities $(1-10 \mathrm{~km})$ are responsible for the perturbations of the wave front, as described in P1. Their effects on star and satellite signals are known as ionospheric scintillations and are studied for their application to satellite transmissions (GLONASS/GPS, Galileo) (e.g. Aarons, 1988). The scintillations are related to the statistical properties of the turbulent ionosphere and are associated to F-region $(150 \mathrm{~km}-500 \mathrm{~km})$ irregularities from several hundreds of meters to kilometers and have been observed over a wide frequency range, from $10 \mathrm{Mhz}$ to $6 \mathrm{GHz}$ (Crane, 1977). 
In this paper, we first review some of the classic methods of random wave propagation and then focus on the Rytov method (Rytov et al., 1989), which enables a statistical characterization of phase perturbation along the ray path by means of the transverse phase structure function. In order to characterize the ionospheric medium in a realistic way, we construct an empirical model of the 3-D electron density spectrum. We then perform a number of simulations and statistically reproduce the results obtained in P1 based on one-dimensional electron density fluctuations spectra deduced from in-situ measurements made with the AUREOL-3 satellite (Béghin et al., 1982). This is the first evidence of a turbulent middle-scale effect on spectral widths measured by SuperDARN radars in the high-latitude ionosphere.

\section{Theoretical approach of wave propagation in random media}

Wave propagation in continuous random media is typically resolved using a statistical description of the wave front characteristics, in terms of phase and amplitude fluctuations. These fluctuations are related to the medium properties and can be described by the refraction index correlation function or by the structure function (Rytov et al., 1989). Usually, studies are divided into two categories: the scattering of a wave from a random medium and the line-of-sight propagation through a random medium. The latter case involves wave propagation through media containing large-scale random inhomogeneities, whose dimensions are large compared to the wavelength. Practically, provided that the fluctuations remain in the range of validity of the weak fluctuation assumption, approximated solutions can be used.

\subsection{Classical methods}

Under the forward scattering assumption, wave propagation in a random medium characterized by a random dielectric constant $\epsilon_{r}=<\epsilon_{r}>\left[1+\widetilde{\epsilon_{r}}(\boldsymbol{r})\right]$ can be described by the scalar Helmholtz Eq. (1) (e.g. Tatarskii, 1961)

$$
\left(\nabla^{2}+k^{2}\right) u(\boldsymbol{r})=-k^{2} \widetilde{\epsilon_{r}}(\boldsymbol{r}) u(\boldsymbol{r}) .
$$

In Eq. (1), $k$ is the mean wave vector in the medium, $\widetilde{\epsilon_{r}}(\boldsymbol{r})$ is the relative fluctuation of the dielectric permittivity. In general, Eq. (1) has no exact solution but it may be solved using a perturbation series in the case of weak fluctuations. This can be done in two ways. The Born approximation consists of expanding the solution $u$ in a series $u=u_{0}+u_{1}+u_{2}+\ldots$, where $u_{0}$ is the wave field in absence of perturbations and the following terms are the perturbations at different orders. The Rytov method consists of writing the solution as a product of different order terms, as $u=u_{0} \cdot u_{1} \cdot u_{2} \ldots$ The method relies on the expression of fields as $u(\boldsymbol{r})=u_{0}(\boldsymbol{r}) \cdot e^{\Phi(\boldsymbol{r})}$, where $u_{0}(\boldsymbol{r})$ is a reference field, usually the wave field without perturbations, and $\Phi(\boldsymbol{r})$ is the complex phase. The solution can then be expressed as $u=u_{0} . e^{\Phi_{0}+\Phi_{1}+\Phi_{2}+\ldots}$ and the problem then reduces to the calculation of the complex phase.
Generally, both methods are performed at the first order, under the weak fluctuations assumption, i.e. when relative dielectric fluctuations are small. However, it is generally agreed (Crane, 1977; Ishimaru, 1978) that the weak fluctuation solution for the phase fluctuation given by the Rytov method is valid beyond the range of validity of the solution for the amplitude fluctuation. In particular, the Rytov solution for the phase solution is valid even in the case of strong fluctuations, where the solution for the amplitude fluctuation is no longer valid.

The observation of ionospheric scintillations has led a number of authors to use these methods in order to determine the statistical characteristics of waves propagating through the ionosphere, for example, Budden (1965) for the Born method or Chytill (1970), Wernik and Liu (1974) for the Rytov method. However, the most common technique is the phase screen method, described in detail by Ratcliffe (1956) and Mercier (1962). The phase screen method consists of modeling the ionosphere as a thin layer or a set of layers representing the ionospheric statistical properties. After crossing these screens, the phase of the incident wave is perturbed and the problem reduces to classical diffraction. This method has been intensively used in the study of natural and artificial sources propagating through the ionosphere in an effort to described ionospheric turbulence properties (e.g. Briggs and Parkin, 1963; Singleton, 1974; Booker and Tao, 1987; Wagen and Yeh, 1989). The phase screen method is well adapted to the case of wave propagating transversely through the ionospheric layers. For SuperDARN HF radars, refraction is used to achieve perpendicularity to the Earth's magnetic field above auroral zones (Villain et al., 1984), so that the propagation through the ionosphere is mainly oblique, except at the far end of the ray path. In this paper, we will then apply the Rytov method rather than the phase screen method to describe the propagation, but we will ignore largescale refraction effects.

\subsection{The Rytov method}

The Rytov method or method of smooth perturbations (MSP) has been described in detail in Rytov et al. (1989). It is a perturbative method for solving the parabolic equation derived from the Helmholtz equation and is valid under the forward scattering assumption, i.e. in the case of inhomogeneities large compared to the wavelength, in particular when large-angle scattering and especially backscattering can be neglected. The solution may be expressed in the form of Eq. (2), where the time dependence of $\exp (-i \omega t)$ is assumed and where $\rho$ is the position in the plane transverse to the propagation which occurs in the z-direction and $A_{0}$ is the initial wave amplitude. The imaginary part of the complex phase $S^{\prime}(\rho, z)=S(\rho, z)-k z$ is the deviation of the phase from its regular shift $k z$ in a medium without inhomogeneities and the real part is the log-amplitude fluctuation

$u(\boldsymbol{\rho}, z)=A_{0} e^{i S^{\prime}(\boldsymbol{\rho}, z)+\ln \frac{A(\rho, z)}{A_{0}}} e^{\mathrm{ikz}}$. 
Expressing the complex phase in terms of its Fourier components and expanding the phase as a series of perturbations, and under the assumption of a statistically homogeneous and isotropic medium, Rytov et al. (1989) express the phase transverse structure function in the form of Eq. (3)

$D_{S}(\boldsymbol{\rho}, z)=\left\langle\left[S^{\prime}\left(\boldsymbol{\rho}^{\prime}, z\right)-S^{\prime}\left(\boldsymbol{\rho}+\boldsymbol{\rho}^{\prime}, z\right)\right]^{2}\right\rangle$

$J_{0}(\kappa \rho)\left(1+\frac{k}{\kappa^{2} z} \sin \frac{\kappa^{2} z}{k}\right) \Phi \epsilon(\kappa, 0) \kappa d \kappa$.

In Eq. (3), $J_{0}$ is the first order Bessel function and $\Phi_{\epsilon}(\kappa, k z)$ is the three-dimensional spectrum of permittivity fluctuations, expressed in cylindrical coordinates. For forward scatter, only the zero frequency term $(k z=0)$ is needed.

\subsection{Doppler enhancement of spectral widths}

Ionospheric inhomogeneities result in amplitude and phase fluctuations of the wave front. The aim of this paper is to estimate the impact of these fluctuations on spectral widths and to compare them to values deduced from radar measurements presented in P1. The primary data product of SuperDARN radars is the temporal autocorrelation function (ACF) of the backscattered signal received from a radar cell, whose typical dimension is of the order of $50 \mathrm{~km}$. Amplitude fluctuations of the waves received by the radars certainly contribute to increase the noise level but will not induce any major effect on the temporal decorrelation of the ACF. In theory, the spectral width is only related to the distribution of turbulent velocities of the scatterers. The amplitude effect on the ACF and on the Doppler spectrum can thus be neglected. On the contrary, phase fluctuations will result in fluctuations of the angle-ofarrival of the wave front arriving on scattering zones. Rytov et al. (1989) or Ishimaru (1978) have shown that the statistical variance of the fluctuation of the angle-of-arrival in the plane transverse to propagation is related to the transverse phase structure function through Eq. (4)

$<\theta^{2}>=\lim _{\rho \rightarrow 0} \frac{D_{S}(\rho)}{k^{2} \rho^{2}}$.

The waves transmitted by SuperDARN radars propagate through ionospheric turbulent layers in order to achieve perpendicularity to magnetic field lines, where the signal is backscattered and Doppler shifted by the inhomogeneities contained in the sounded volume (radar cell). For a wave front arriving on a single moving irregularity, the Doppler shift depends on the irregularity velocity $\boldsymbol{V}$ and on the angle of arrival $\theta$. An ideal unperturbed wave front will result in a delta function Doppler spectrum, as illustrated on Fig. 1.

A perturbed wave front will result in a distribution of Doppler shifts and thus gives rise to an enhancement in spectral width. The spectral width thus depends on two factors: the turbulent velocity distributions of inhomogeneities in the sounded volume, and the distribution of angles of arrival of the wave front on each inhomogeneity. Spectral width values given by SuperDARN radars are a composite of both contributions.

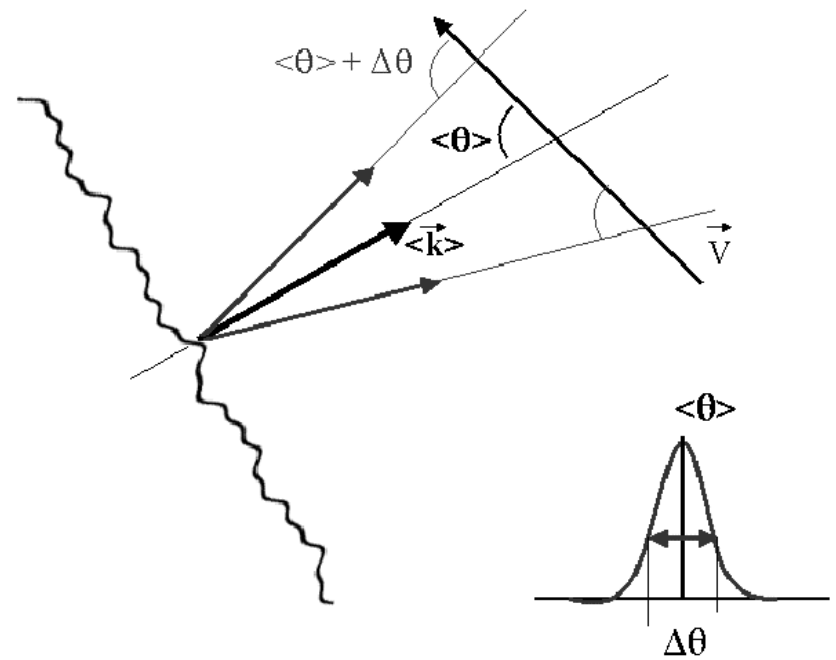

Fig. 1. Effect of the fluctuations of the angle-of-arrival on the Doppler spectrum. The wave front arrives on an inhomogeneity moving with the velocity $\boldsymbol{V}$ with a mean angle $\langle\theta>$. The fluctuation of the phase gives rise to a distribution of angles-of-arrival characterized by $\Delta \theta$.

Transforming spectral width values in terms of velocity, the shift due to phase perturbation may be expressed by Eq. (5), where $\theta$ is the perturbed angle of arrival and $\langle\theta>$ is the mean arrival angle

$\delta W_{<W>}=|\boldsymbol{V}|(\cos \theta-\cos <\theta>)$.

The distribution of $\delta W$ terms in Eq. (5) represents the contribution of phase perturbations to the total spectrum. For a sufficiently large wave front (as compared to the spatial scale of the inhomogeneity), the angle of arrival distribution can be considered as a gaussian. In this case, the spectrum half width is given by Eq. (6), where $<\delta W^{2}>^{1 / 2}$ is the standard deviation of the Doppler shift distribution.

$\Delta W=2 \sqrt{2 \ln 2}<\delta W^{2}>^{1 / 2}$.

In their standard operating modes, the SuperDARN radars use this quantity to estimate the spectral width value. Spectral widths are thus biased due to the decorrelation effect of the wave front as it propagates. Using the Rytov method, this effect can be quantified via the phase structure function and the angle-of-arrival variance calculation. The spectral enhancement due to the decorrelation will thus depend on the permittivity spectrum and on the propagation distance.

The Rytov method leads to an estimation of the variance of the angle of arrival that characterizes the decorrelation of the wave front as it propagates. As seen in the previous section, this loss of coherence results in an overestimation of the spectral width measured by the SuperDARN radars. This enhancement depends of the statistical properties of the medium through the 3-D permittivity spectrum. This spectrum can be deduced from the 1-D spectrum obtained, for instance by satellite measurements of the electron density. In 


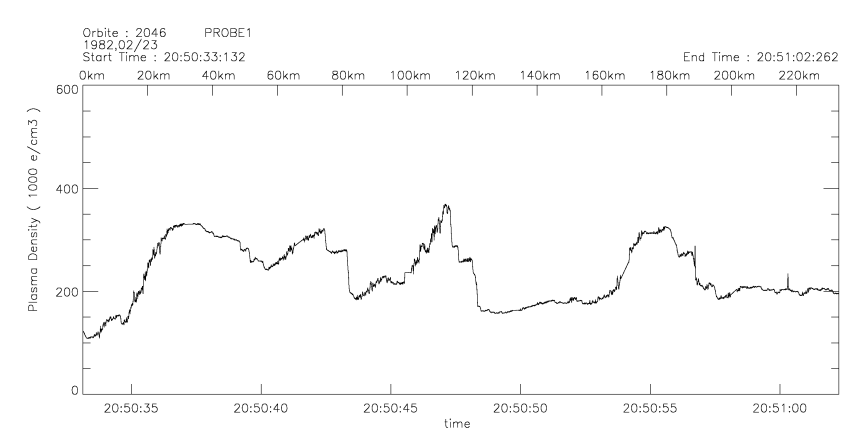

Fig. 2. Electron density profile extracted from ISOPROBE data, along the satellite path, for a 23 February 1982 orbit.

the following section, we estimate a realistic spectrum of the permittivity fluctuations, based upon the AUREOL-3 satellite data.

\section{Determination of the ionospheric fluctuation spec- trum}

In this section, we use satellite data in order to estimate a permittivity spectrum suitable to the Rytov method and thus adapted to the SuperDARN configuration. In a first part, we derive the 1-D spectrum along the satellite trajectory, and then estimate the 3-D spectrum at high latitudes. Using several data sets, we estimate a semiempirical 3-D spectrum model which will be the input in numerical simulations based upon the Rytov method.

\subsection{Data set}

The ISOPROBE experiment (Béghin et al., 1982), on board the AUREOL-3 satellite (1981-1986), gives access to the plasma frequency in the auroral zones at altitudes in the range $400 \mathrm{~km}-600 \mathrm{~km}$ with a very high spatial resolution of $8 \mathrm{~m}$. This is only slightly higher than the altitudes of radar measurements. In the auroral zones, inhomogeneities are strongly field-aligned, allowing one to consider that they are mapped to lower altitudes and that the satellite data will realistically reproduce the statistical properties of the medium sounded by the radars. As seen in the previous section, those inhomogeneities that affect the spectral width values are large as compared to the wavelength but are limited by the radar cell typical scale $(50 \mathrm{~km})$. Usual frequencies used by SuperDARN radars lead to wave backscattering by $15-\mathrm{m}$ inhomogeneities, so that the ISOPROBE spatial resolution is sufficiently high to describe spatial scales of interest in this study.

Figure 2 shows an example of electron density measured by ISOPROBE on orbit 2046 (23 February 1982) for a $230 \mathrm{~km}$ path of about $30 \mathrm{~s}$. The upper scale is the distance along the satellite path and the lower scale is time. The mean electron density is $210^{11} \mathrm{~m}^{-3}$, leading to a mean plasma frequency of the order of $4 \mathrm{MHz}$. Figure 2 clearly shows

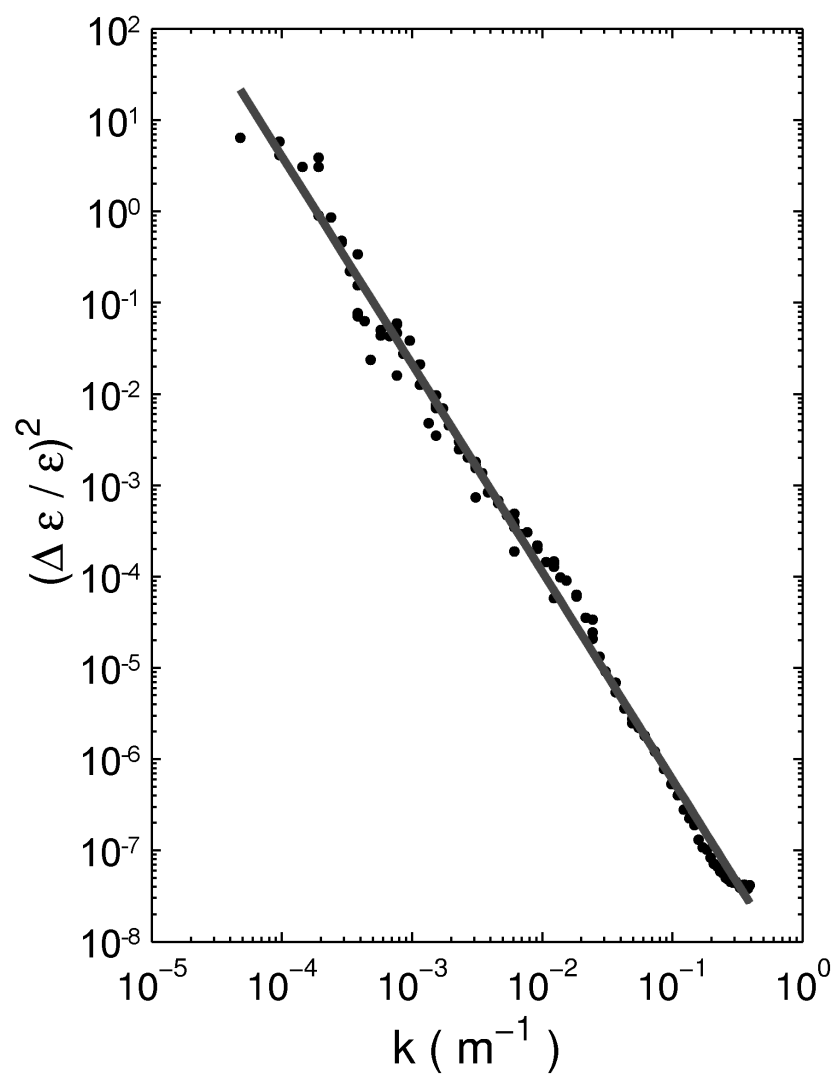

Fig. 3. Permittivity fluctuations spectrum (dots) and power-law fit (line) giving a slope of -2.27 .

large-scale structures, of about $20 \mathrm{~km}$ long, where the electron density rises by a factor of 1.5 to 2 , especially around 20:50:47 and 20:50:55 UT. These structures represent largescale ionospheric inhomogeneities, and give rise to smaller scale inhomogeneities mainly through instability processes and turbulent cascade. Cerisier et al. (1985) have shown that the major cascading mechanism in these regions is the gradient drift instability and that it explains the differences observed between upward gradients showing a high-level of perturbation and stable downward gradients where the instability is inoperative.

\subsection{Estimation of 1-D spectrum}

We have selected a set of 23 orbits representing the typical fluctuations encountered by AUREOL- 3 in the polar regions. For each of these orbits, the permittivity has been computed using a wave frequency $f_{r}$ (radar frequency) ranging from $5 \mathrm{MHz}$ to $20 \mathrm{MHz}$ by steps of $0.5 \mathrm{MHz}$. The mean plasma frequency $f_{p}$ is also estimated to obtain a value of $X=\frac{f_{p}}{f_{r}}$. The spectrum of electric permittivity $\epsilon_{r}=1-X^{2}$ is then computed using the Welch method (Welch, 1967).

Figure 3 shows an example of a 1-D spectrum obtained from ISOPROBE data as a function of the components of the Fourier space $\kappa\left(\mathrm{m}^{-1}\right)$, between $10^{-5} \mathrm{~m}^{-1}$ and $10^{-1} \mathrm{~m}^{-1}$. In this case, $X=0.48$. In first approximation, the 1-D spectrum 
can be seen as a power law spectrum with a -2 slope, as typically encountered in the literature (Rufenbach, 1972; Dyson et al., 1974). The continuous line on Fig. 3 represents the linear fit of the estimated spectrum and leads to a slope equal to -2.27 .

Following Woodman and Basu (1978), we consider a 1D spectrum of the form of Eq. (7), which is very close to a -2 slope power law with a cut-off at large-scales. This cutoff is determined by the $L_{0}$ factor which can be seen as the turbulent outer scale

$\Phi(\kappa)=\Phi_{0} \frac{1}{1+\kappa^{2} L_{0}^{2}}$.

In Eq. (7), $\Phi_{0}$ is the amplitude of the spectrum. Eq. (7) enables an analytical fit for the experimental spectrum. Furthermore, under some hypothesis on the geometry of the inhomogeneities, it allows one to deduce analytically the 3-D spectrum.

\subsection{3-D spectrum and calibration}

In the high-latitude F-region, the ionospheric turbulence is confined to the plane transverse to the Earth's magnetic field (Bates and Albee, 1970) and at first order, it can be considered as isotropic in this plane. Under the assumption of infinitely elongated cylindrical inhomogeneities, Woodman and Basu (1978) deduce from Eq. (7) the 3-D spectrum in the form of Eq. (8), where $\kappa_{\|}$is the Fourier component in the field lines direction and $\kappa_{\perp}$ is the component in the transverse plane.

$\Phi(\kappa)=\frac{\Phi_{0} L_{0}}{2} \frac{\delta \kappa_{\|}}{\left(1+\kappa_{\perp}^{2} L_{0}^{2}\right)^{3 / 2}}$.

Figure 4 shows an example of a 1-D permittivity fluctuation spectrum, extracted from a $300 \mathrm{~km}$ long segment of orbit 2086 (26 February 1982). The black line represents the fit to a power law and gives a -2.12 value for the slope. Blue and red lines represent fits following Eq. (9), with either a free $c$ coefficient for the blue line or with $c=2$ for the red fit. For the example given in Fig. 4, the values of coefficients $a, b$ and $c$ are, respectively, $0.72,2.1110^{8}$ and 2.12 for the blue line and $1.12,1.8110^{8}$ and 2 for the red fit

$\Phi(\kappa)=\frac{a}{1+b \kappa^{c}}$.

For all the data sets, the agreement between power law fits and Eq. (9) fits is excellent, with a relative difference between the $c$ parameter and 2 of the order of $2 \%$.

In the example of Fig. $4, L_{0}$ deduced from $b$ is $13.5 \mathrm{~km}$ and can be identified as the outer scale of turbulence (strictly in the case of a $c$ parameter fixed to 2 , as seen above). This value seems to agree with the size of structures observed in Fig. 2. In the data sets considered for the present study, $L_{0}$ ranges from $10 \mathrm{~km}$ to $30 \mathrm{~km}$, and is in agreement with the characteristic dimensions of large-scale inhomogeneities classically encountered in the literature (e.g. Tsunoda, 1988).

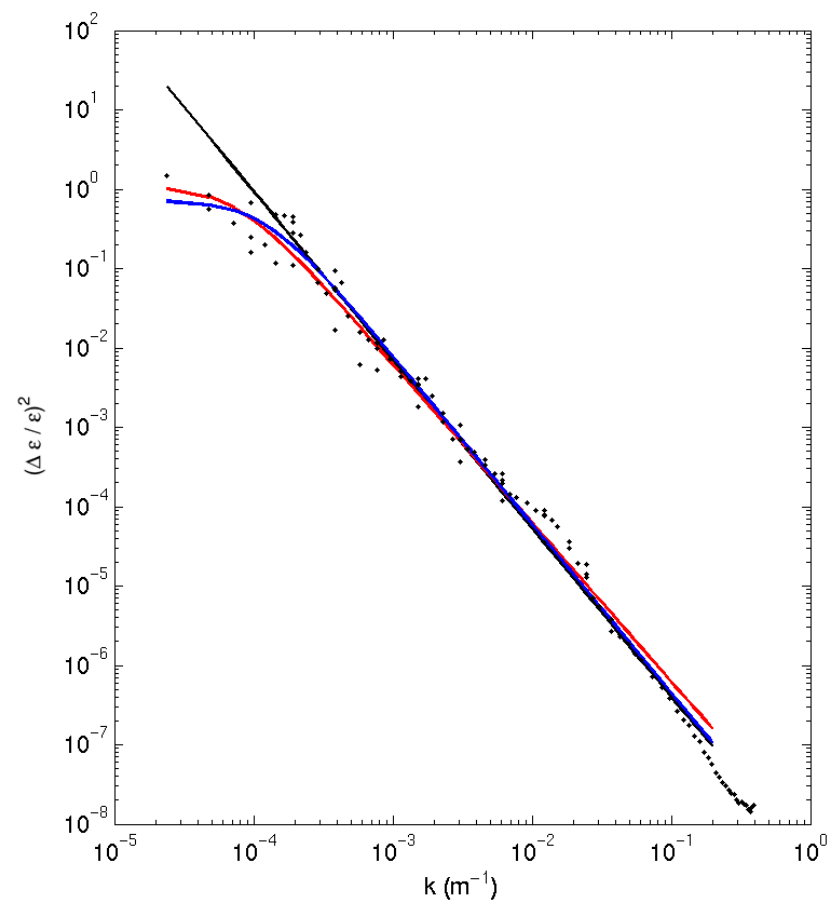

Fig. 4. Example of a 1-D permittivity spectrum (dots), linear fit (black line), fit with a free slope (blue line) and fit with a -2 fixed slope (red line) following Eq. (9).

We have chosen the second fit, with a fixed slope of 2, since it allows one to estimate the 3-D spectrum, as mentioned earlier. Spectra are computed as a function of $X$ using Eq. (9). For each data set, only the amplitude (represented by the $a$ parameter) depends upon $X$. In order to take this variation into account, we have represented the amplitude variation of the spectrum by a 3-order polynomial. We can thus estimate the spectra characteristics as functions of $X$ for each data set. These spectra are supposed to be valid for the range of altitudes sounded by the SuperDARN radars and are used in the numerical simulations described in the following section. In these simulations, refraction effects are neglected and the whole propagation is supposed to be described by the 3-D spectrum calculated in the plane perpendicular to $\boldsymbol{B}$. In other words, no aspect angle dependence is considered, since the wave vector of the radar wave is mostly close to perpendicularity $(<10 \mathrm{deg})$ along the propagation path.

\section{Numerical simulations}

Using the analytical developments of Sect. 1 and the semiempirical spectrum model described in Sect. 2, we have performed a number of numerical simulations in order to estimate the statistical properties of a wave front arriving on a typical SuperDARN sounded cell . Wave fronts are described by $1 E 5$ points. Along the propagation path, the variance of angles of arrival is estimated using the 3-D permittivity spectra calibrated by the value of the $X$ parameter. In order to allow for a direct comparison of these simulations with the 


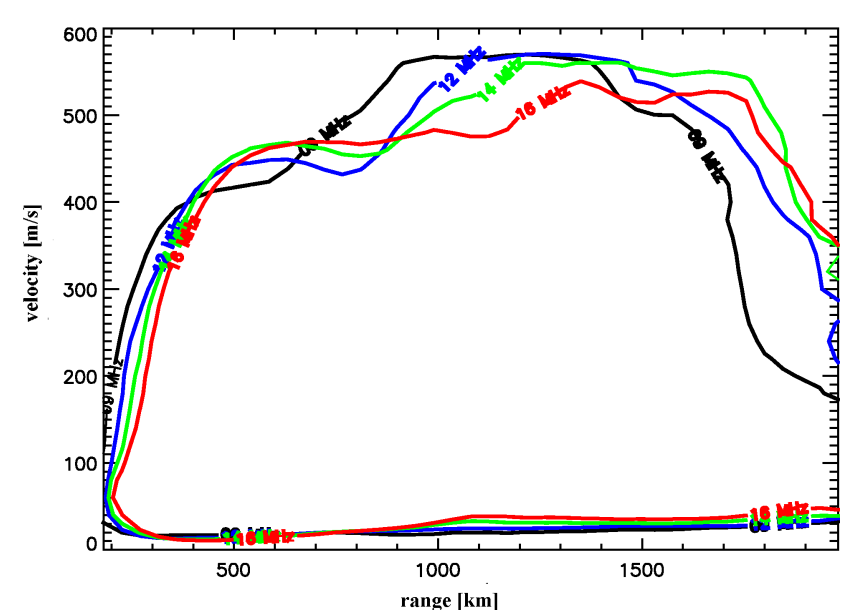

Fig. 5. Range-velocity distribution of echoes associated with the database used for the study described in Vallières et al. (2003). Contours representing a level of $50 \%$ of the occurrence are plotted for $9 \mathrm{MHz}$ (black), $12 \mathrm{MHz}$ (blue), $14 \mathrm{MHz}$ (green) and $16 \mathrm{MHz}$ (red).

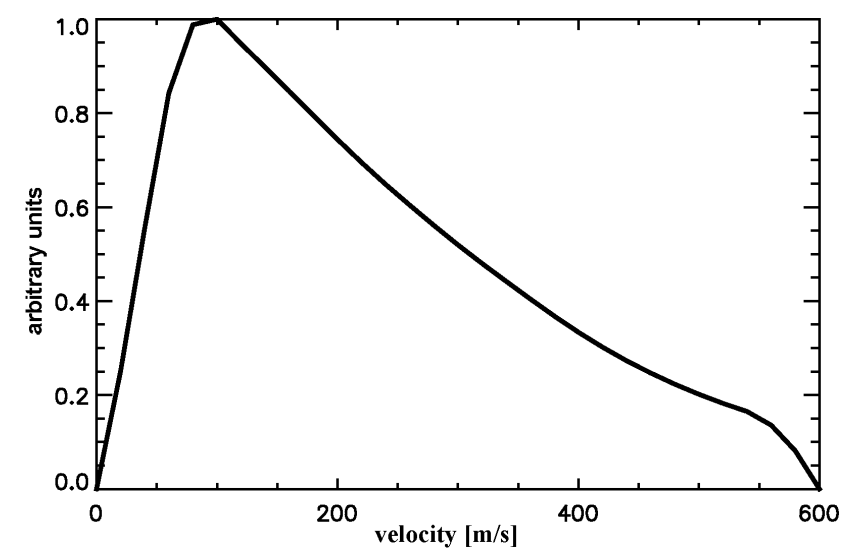

Fig. 6. Normalized mean velocity distribution between 0 and $600 \mathrm{~m} . \mathrm{s}^{-1}$ calculated from the database used in Vallières et al. (2003) and averaged over the 4 radar frequencies and all radar ranges.

results described in $\mathrm{P} 1$, radar frequencies are fixed to values of 9, 12, 14 and $16 \mathrm{MHz}$. Results are estimated at distances between $180 \mathrm{~km}$ and $1000 \mathrm{~km}$. For each radar frequency and each propagation distance, the phase structure function is estimated and the variance of the angle of arrival is deduced. The wave front is then supposed to be scattered by a volume of irregularities described by a typical distribution of turbulent velocities. Figure 5 shows the range-velocity distribution of echoes obtained with SuperDARN radars, for the database described in $\mathrm{P} 1$.

Figure 5 shows the $50 \%$-level contours for the 4 radar frequencies (9 MHz, $12 \mathrm{MHz}, 14 \mathrm{MHz}$ and $16 \mathrm{MHz})$. It shows that the velocity distributions do not depend upon radar frequency and range. Each distribution contains around 450000 points. The velocity distributions show little variation from one range gate to another. Figure 6 shows the normalized mean velocity distribution associated with the database, av-

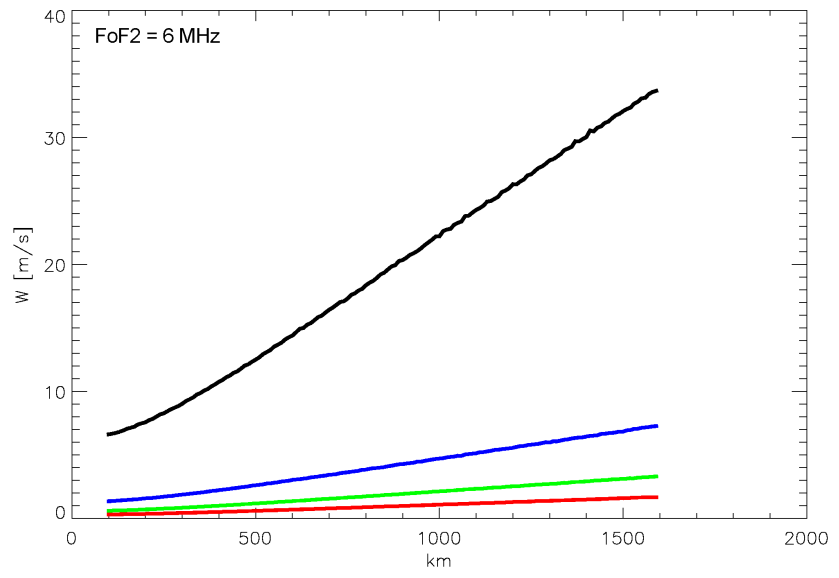

Fig. 7. Results of the simulations for the 2046-orbit extract of Fig. 1 and for a radar frequency of $9 \mathrm{MHz}$ (black), $12 \mathrm{MHz}$ (blue), $14 \mathrm{MHz}$ (green) and $16 \mathrm{MHz}$ (red) and a plasma frequency equal to $6 \mathrm{MHz}$ (bottom right). Results are given in units of spectral width (m.s ${ }^{-1}$ ) as a function of the propagation distance.

eraged over the four frequencies and all range gates. The distribution emphasizes a peak at $100 \mathrm{~m} . \mathrm{s}^{-1}$, a width at half height of the order of $250 \mathrm{~m} . \mathrm{s}^{-1}$ and a strong asymmetry.

In the simulations described here, this distribution is modelled by a gamma law, in order to represent the velocity distribution of inhomogeneities in the volume that scatter the radar wave front. The mean local angle of arrival is chosen randomly in a normal distribution between 0 and $2 \pi$. Each point of the wave front interacts with an irregularity and gives rise to a Doppler shift. The local angle of arrival imposes the velocity value as measured by radars (see Fig. 1) and leads to estimate the shift $\delta W_{<W>}$ (Eq. 5) due to the wave front decorrelation. The distribution of these shifts over the whole scattering volume gives the spectral enhancement due to propagation.

\subsection{Case of a statistically homogeneous turbulence}

Figure 7 shows the results of our simulations for an extract of orbit 2046 and a mean plasma frequency arbitrarily fixed to $6 \mathrm{MHz}$. In this first set of simulations, we consider a statistically homogeneous turbulent medium. In other words, the wave front propagates in an ionosphere whose mean plasma frequency is constant over the propagation and where the decorrelation only depends on the distance. The spectral enhancement due to decorrelation is given in $\mathrm{m} . \mathrm{s}^{-1}$ as a function of the propagation distance in the medium for the four radar frequencies used in P1. As seen in Fig. 7, spectral width values rise linearly with the distance and this effect is highly nonlinear with the radar frequency. For higher frequencies, the decorrelation effect is low and the spectral width enhancement is of the order of $15 \mathrm{~m} . \mathrm{s}^{-1}$ for $14 \mathrm{MHz}$ and $16 \mathrm{MHz}$ at $1000 \mathrm{~km}$. At $12 \mathrm{MHz}$, the decorrelation is more efficient and the spectral enhancement rises to $25 \mathrm{~m} . \mathrm{s}^{-1}$ and at $9 \mathrm{MHz}$; the increase in spectral width can reach values 
as high as $120 \mathrm{~m} . \mathrm{s}^{-1}$ at $1000 \mathrm{~km}$. The shift between $9-\mathrm{MHz}$ values and $16-\mathrm{MHz}$ values and the variation in frequency is related to the nonlinear dependence of the spectrum amplitude with the plasma frequency to radar frequency ratio.

The results of the simulations largely depend upon the spectrum amplitude associated with each orbit extract. In order to reproduce a mean effect, 23 orbits representing typical high-latitude ionospheric fluctuations were selected. The mean results are very close to those of Fig. 7 but the standard deviation can be important. However, results show that, on average, spectral widths between $9 \mathrm{MHz}$ and $16 \mathrm{MHz}$ can differ from $100 \mathrm{~m} . \mathrm{s}^{-1}$ due to the decorrelation at distances as far as $1000 \mathrm{~km}$.

Figure 7 shows a frequency organisation coherent with the experimental results presented in P1. Moreover, for far ranges, the $9 \mathrm{MHz}$ curve seems to level off, revealing a saturation process. This behavior has also been described in $\mathrm{P} 1$.

\subsection{Influence of vertical ionospheric electron density pro- file}

In the previous section, the mean plasma frequency was considered to be constant all along the propagation path. In order to approach the conditions of propagation encountered in the ionosphere, we now include a vertical dependance of the plasma frequency and introduce a simple straight line propagation model, in order to estimate the range associated with echoes observed by SuperDARN radars. The vertical dependance of plasma frequency is modelled by a classic Chapman layer between $100 \mathrm{~km}$ and $300 \mathrm{~km}$, with a peak at $300 \mathrm{~km}$ and a $80-\mathrm{km}$ scale height. The simulations have been performed for a maximum electron density of $6 \mathrm{MHz}, 7 \mathrm{MHz}$ and $8 \mathrm{MHz}$, respectively. With the typical parameters used by SuperDARN radars, ionospheric echoes are obtained at altitudes just below the electron density maximum. In this study, we fixed the altitude of scattering zones to $280 \mathrm{~km}$. Results are computed for typical radar ranges, i.e. between $180 \mathrm{~km}$ and $1600 \mathrm{~km}$. For each range considered, we estimate the elevation angle needed to attain the supposed scattering altitude, taking the Earth's curvature into account. For each range, we thus estimate the propagation distance in the ionosphere and the variation of plasma frequency along the wave front path.

The permittivity variation along the ray path is taken into account in the Rytov method by considering that the amplitude of the spectrum $a\left(z^{\prime}\right)$ varies with $z^{\prime}$. In particular, this assumption leads to neglect the variations of the term $\left(1+\frac{k}{\kappa^{2} z} \sin \frac{\kappa^{2} z}{k}\right)$ in Eq. (3). By continuously summing over a succession of $d z$-wide layers, one can then obtain the expression (10) of the transverse phase structure function, where $\widetilde{\Phi}_{\epsilon}$ is the normalized spectrum and $a\left(z^{\prime}\right)$ is its amplitude at the altitude $z^{\prime}$ :

$$
D_{S}(\rho, z)=\pi^{2} k^{2} \int_{0}^{z} a\left(z^{\prime}\right) d z^{\prime}
$$
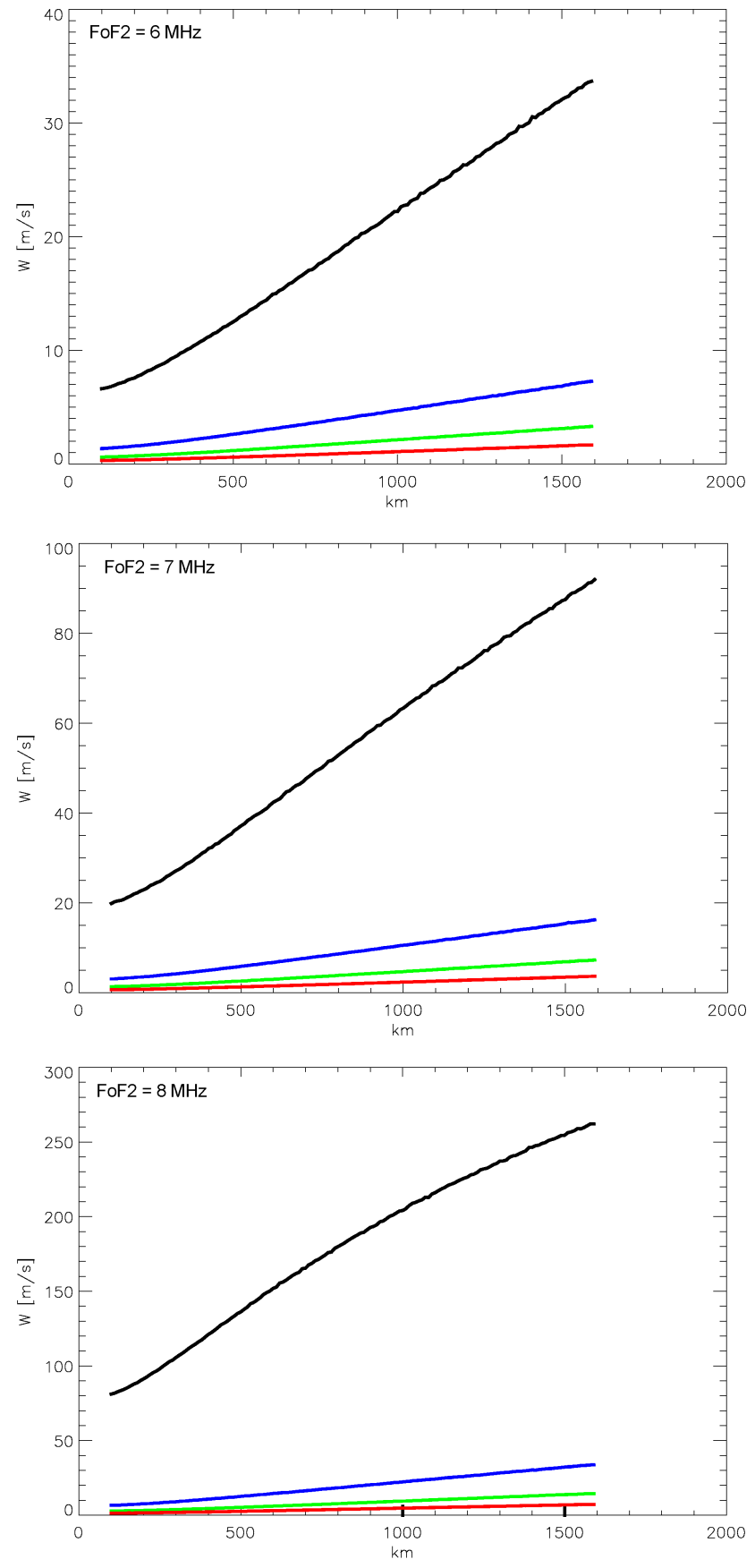

Fig. 8. Spectral width enhancement due to decorrelation, averaged over 23 orbits for a radar frequency of $9 \mathrm{MHz}$ (black), $12 \mathrm{MHz}$ (blue), $14 \mathrm{MHz}$ (green), $16 \mathrm{MHz}$ (red) and a maximum plasma frequency of $6 \mathrm{MHz}$ (top), $7 \mathrm{MHz}$ (middle) and $8 \mathrm{MHz}$ (bottom).

$\int_{0}^{\infty}\left[1-J_{0}(\kappa \rho)\right]\left(1+\frac{k}{\kappa^{2} z} \sin \frac{\kappa^{2} z}{k}\right) \widetilde{\Phi}_{\epsilon}(\kappa, 0) \kappa d \kappa$

Figure 8 shows the results of the simulations, color-coded as before for the radar frequencies, averaged over the 23-orbit extracts, for plasma frequency peaks of $6 \mathrm{MHz}$ (top), $7 \mathrm{MHz}$ (middle) and $8 \mathrm{MHz}$ (bottom) and for radar distances ranging from $180 \mathrm{~km}$ to $1600 \mathrm{~km}$, to allow for direct comparison 
to experimental results presented in P1. These simulations are more realistic since they give values close to those mentioned in P1. The bottom plot shows values large as compared to experimental results and is given here as an illustration of the far range saturation process clearly evidenced in $\mathrm{P} 1$. The decorrelation effect leads to spectral width enhancements of $30 \mathrm{~m} . \mathrm{s}^{-1}$ for a $6-\mathrm{km}$ peaking ionosphere and of $60 \mathrm{~m} . \mathrm{s}^{-1}$ for $7 \mathrm{~km}$ at $1500 \mathrm{~km}$. In P1, radar data show a shift in spectral width values up to $50 \mathrm{~m} . \mathrm{s}^{-1}$ between echoes obtained at $9 \mathrm{MHz}$ and those obtained at $16 \mathrm{MHz}$ at far ranges. These simulations thus reproduce the order of magnitude of the spectral width enhancement due to decorrelation and the behaviour of data distributions obtained at different radar frequencies. Even at low ranges, the effect for a $9-\mathrm{MHz}$ radar frequency is not negligible. This point seems to disagree with the results of P1. Indeed, the frequency effect has been shown to affect F-region echoes but not E-region echoes. The difference between the results of these simulations and the results described in P1 can be explained by the fact that we consider that all echoes are generated at the same altitude. More realistic simulations should take into account large-scale refraction effect. Radars waves with a frequency close to the plasma frequency will be refracted more efficiently and can reach perpendicularity to the Earth's magnetic field at lower altitudes and nearer ranges. The propagation model considered here is too simple to reproduce all the features observed on the spectral width distributions. However, the simulations still reproduce the effect of meso-scale inhomogeneities.

Even if there can be others factors influencing the spectral width value obtained at different radar frequencies, the effect of decorrelation due to meso-scale electron irregularities is shown to be mainly responsible for the difference observed in the distribution of SuperDARN spectral widths as a function of transmitted frequency.

\section{Discussion and conclusion}

Previous statistical studies have shown the existence of a frequency effect in the range distributions of spectral widths determined at several frequencies by radars from the SuperDARN network. At distances as far as $1500 \mathrm{~km}$, the shift between spectral width measured at $9 \mathrm{MHz}$ and measured at $16 \mathrm{MHz}$ can be as high as $50 \mathrm{~m} \cdot \mathrm{s}^{-1}$.

In this paper, this effect is interpreted as the consequence of the loss of coherence of the wave front as it propagates through a turbulent ionosphere. This decorrelation is explained in terms of an interaction between the wave front and the inhomogeneous ionosphere along the propagation path. The meso-scales structures decorrelate the incident wave front, depending on the radar and plasma frequencies and on the propagation distance. The modelling of this effect is conducted using the Rytov method and the model is implemented with realistic semiempirical permittivity spectra derived from AUREOL-3 satellite measurements. The model also takes into account a simple propagation model and a classic ionospheric plasma density model. The spectral en- hancement due to propagation is then related to the statistical deformation of the wave front impinging on a typical volume of ionospheric irregularities.

The numerical simulations show that the amplitude of the permittivity spectrum is a parameter of prime importance and determine mainly the value of the spectral width enhancement due to decorrelation. Thus, at far ranges, the shift between values obtained at $9 \mathrm{MHz}$ and those obtained at $16 \mathrm{MHz}$ reach $30 \mathrm{~m} \cdot \mathrm{s}^{-1}$ for a plasma frequency peak of $6 \mathrm{MHz}$ and $90 \mathrm{~m} . \mathrm{s}^{-1}$ when the peak is at $7 \mathrm{MHz}$. The model reproduces the behavior of the spectral width distributions and shows that the middle-scale interactions are responsible for the shift observed in the spectral width values measured at different frequencies.

More realistic simulations should take into account a variety of propagation and ionospheric models representing the very varied conditions of sounding by SuperDARN radars. However, propagation models involve large-scale interaction and the purpose of this study is to emphasize the role of middle-scale irregularities. The increase in the number of models can make the quantitative interpretation of the results difficult and the consideration of more realistic models is somewhat more suitable for case studies. This kind of consideration leads us to only consider quite simple models, in order to observe the sole effect of the wave front decorrelation.

However, this study evidences the role of wave front decorrelation over the spectral width determination by SuperDARN radars and shows a systematic bias in the spectral width values obtained at low frequencies as compared to those obtained at higher frequencies. This effect has to be taken into account in the numerous geophysical studies performed with SuperDARN spectral widths, especially with the increasing implication of SuperDARN radars in space weather. In particular, the determination of geophysical regions based upon large databases mixing spectral widths obtained at night and day frequencies has to be taken carefully into account for the understanding of exchange processes in the field of Sun-Earth connections.

Acknowledgements. The authors thank Vladimir Krasnosselskikh for helpful discussions. Thanks are also due to the French and Russian staff for the operation of the AUREOL-3 satellite.

Topical Editor M. Lester thanks two referees for their help in evaluating this paper.

\section{References}

Aarons, J.: Global Positioning System Phase Fluctuations at Auroral Latitudes, Geophys. Res. Lett., 102, 17 219-17 231, 1988.

Bates, H. F. and Albee, P. R.: Aspect sensitivity of F-layer HF backscatter echoes, J. Geophys. Res., 75, 165-170, 1970.

Béghin, C., Karczewski, J. F., Poirier, B., Debrie, R., and Massevitch, N.: The ARCAD-3 ISOPROBE experiment for high time resolution thermal plasma measurements, Ann. Geophysicae, 38, 615-629, 1982. 
Booker, H. G. and Tao, J. W.: A scintillation theory of HF waves returned from the F-region: receiver near transmitter, J. Atmos. Terr. Phys., 49, 915-938, 1987.

Briggs, B. H. and Parkin, I. A.: On the variation of radio star and satellite scintillations with zenith angle, J. Atmos. Terr. Phys., 25, 339-365, 1963.

Budden, K. G.: The amplitude fluctuations of the radio wave scattered from a thick ionospheric layer with weak irregularities, J. Atmos. Terr. Phys., 27, 155-172, 1965.

Cerisier, J. C., Berthelier, J. J., and Béghin, C.: Unstable density gradients in the high-latitude ionosphere, Radio Sci., 20, 755761,1985 .

Chytill, B.: Amplitude and phase scintillations of spherical waves, J. Atmos. Terr. Phys., 32, 961-966, 1970.

Crane, R. K.: Ionospheric scintillation, Proc. IEEE, 65, 180-199, 1977.

Dyson, P. L., McClure, J. P., and Hanson, W. B.: In situ measurements of the spectral characteristics of F-region ionospheric irregularities, J. Geophys. Res., 79, 1497-1502, 1974.

Ishimaru, A.: Wave Propagation and Scattering in Random Media, vol. 1 and 2, Academic, New York, 1978.

Mercier, R. P.: Diffraction by a screen causing large random phase fluctuations, Proc. Cambridge Phil. Soc., 59, 382-400, 1962.

Ratcliffe, J. A.: Some aspects of diffraction theory and their application to the ionosphere, Rep. Prog. Phys., 19, 190-263, 1956.

Rufenbach, C. L.: Power law wavenumber spectrum deduced from ionospheric scintillation observations, J. Geophys. Res., 77, 4761, 1972.
Rytov, S. M., Kravtsov, Y. A., and Tatarskii, V. I.: Principles of statistical radiophysics, vol. 4, Wave propagation through random media, Springer-Verlag, 1989.

Singleton, D. G.: Power spectra of ionospheric scintillations, J. Atmos. Terr. Phys., 36, 113-133, 1974.

Tatarskii, V. I.: Wave propagation in a turbulent medium, McGrawHill, 1961.

Tsunoda, R. T.: High-latitude F-region irregularities : A review and synthesis, Rev. Geophys., 26, 719-760, 1988.

Vallières, X., Villain, J.-P., and André, R.: Characterization of frequency effect in SuperDARN spectral width distributions, Radio Sci., 38(1), 1003, doi:10.1029/2001RS002550, 2003.

Villain, J.-P., Greenwald, R. A., and Vickrey, J. F.: HF ray tracing at high latitudes using measured meridional electron density distributions, Radio Sci., 19, 359-374, 1984.

Wagen, J. F. and Yeh, K. C.: Simulation of HF propagation and angle of arrival in a turbulent ionosphere, Radio Sci., 24, 196208, 1989.

Welch, P. D.: The use of Fast Fourier Transform for the estimation of power spectra: A method based on time averaging over short, modified periodograms, IEEE Trans. Audio Electroacoust., AU 15, 70-73, 1967.

Wernik, A. W. and Liu, C. H.: Ionospheric irregularities causing scintillation of $\mathrm{GHz}$ frequency radio signals, J. Atmos. Terr. Phys., 36, 871-879, 1974.

Woodman, R. F. and Basu, S.: Comparison between in situ measuremenys of F-region irregularities and backscatter observations at 3-m wavelength, Geophys. Res. Lett., 5, 869-872, 1978. 